\title{
Aportaciones del fútbol en la formación infantil y juvenil en una comunidad vulnerable Soccer contributions to child and youth training in a vulnerable community \\ *Hernán Rivas-Martínez, ${ }^{* *}$ Josefina Bailey-Moreno \\ Corporación Universitaria Minuto de Dios (Colombia), **Tecnologico de Monterrey (México)
}

\begin{abstract}
Resumen. Esta investigación tuvo como propósito conocer la manera en la que la práctica del fútbol contribuye en la educación de los niños, niñas y jóvenes pertenecientes a la Escuela Juego Limpio, Fútbol, Paz y Convivencia. Esta escuela se localiza en el municipio de Garzón -Huila, en Colombia. La población carece de condiciones económicas óptimas para tener una vivienda digna, lugares de sano esparcimiento, y se encuentra expuesta a diversos riesgos psicosociales. En el estudio participaron diez alumnos de ocho a 15 años, de los grados pony, pre-infantil e infantil, cinco madres de alumnos y cinco docentes. Desde un enfoque cualitativo se recolectaron datos mediante observación participante y entrevistas. La aplicación de estas técnicas permitió obtener información de las percepciones y vivencias de los participantes, las cuales fueron analizadas y organizadas en dos categorías: fortalecimiento del crecimiento personal y académico, y transformación del proyecto de vida. Los resultados indican que el fútbol ayuda a la formación integral de los niños, niñas y jóvenes, potencializa cualidades y destrezas, fomenta cambios en los comportamientos, mejora las relaciones interpersonales, genera espacios de esparcimiento y diversión acompañados de procesos académicos que estimulan el interés de ir transformando sus ambientes a través de la creación de proyectos de vida.
\end{abstract}

Palabras clave: Estudiantes en riesgo psicosocial, deporte educativo, desarrollo intelectual, socialización, programa de acción comunitario.

\begin{abstract}
The purpose of this research was to understand the way in which soccer practice contributes to the education of children and young people who belonged to the School Fair Play, Soccer, Peace and Coexistence [Escuela Juego Limpio, Fútbol, Paz y Convivencia]. This school is located in San Felipe, in the municipality of Garzón-Huila, in Colombia. The population lacks of the optimal economic conditions to have decent housing and places of healthy recreation, and is exposed to various psychosocial risks. The study involved six boys and four girls from the pony, pre-childhood and children's grades, five mothers, and five teachers. From a qualitative approach, data was collected through participant observation and interviews. The application of these techniques allowed obtaining information on oarticipants' perceptions and experiences, which were analyzed and organized into two categories: promoting personal and academic growth, and transforming life project. The results suggest that soccer helps the integral formation of children and young people, strengthen qualities and skills, encourages change in behaviors, improves interpersonal relationships, generates recreational and fun spaces accompanied by academic processes that stimulate interest in transforming their environments through the creation of life projects.
\end{abstract}

Keywords: Students at psychosocial risk, educational sport, intellectual development, socialization, community action programme.

\section{Introducción}

Este artículo presenta los resultados de una investigación que se llevó a cabo en la Escuela de Fútbol Juego Limpio: Fútbol, Paz y Convivencia (en adelante la Escuela), proyecto que ha centrado su labor social en un asentamiento irregular del municipio de Garzón-Huila, Colombia (Alcaldía de Garzón, 2016). La Escuela que tiene como propósito el aprovechamiento del tiempo libre y el fortalecimiento de los proyectos de vida de niños, niñas y jóvenes (en adelante los alumnos) cuenta con cuatro categorías deportivas: baby, pony, pre-infantil e infantil. También se realizan actividades académicas para reforzar la lectura, las matemáticas y los valores, con la intención de complementar la formación educativa impartida en los colegios a los que asisten los alumnos. Los horarios en la Escuela son de 7:00 am a 11:00 am y de 2:00 pm a 5:00 pm, de lunes a viernes, exceptuando días festivos o feriados. La Escuela cuenta con un educador físico quien a través de su planificación entrena en la disciplina a los alumnos. Asimismo, cuenta con siete docentes de apoyo académico quienes realizan trabajo voluntario.

El asentamiento del municipio de Garzón-Huila, surgió a partir del desplazamiento de las familias que anteriormente habitaban la zona (territorios inundados) donde ahora se encuentra la represa de la central hidroeléctrica El Quimbo.

Fecha recepción: 20-03-20. Fecha de aceptación: 25-06-20

Josefina Bailey-Moreno

josefina.bailey@tec.mx
Estas familias solventaban sus gastos a través de la agricultura de tabaco, maíz y producción de otros productos perecederos. Dado que estas familias se quedaron sin sus posesiones, decidieron desplazarse al municipio de Garzón y ubicar sus viviendas en un terreno a las afueras del municipio. Los habitantes se encuentran expuestos a riesgos psicosociales asociados al medio hostil donde conviven al ser miembros de la población víctima del conflicto en Garzón (Registro Único de Víctimas, 2018).

En el asentamiento viven cerca de 345 familias que luchan por sobrevivir ante las dificultades económicas y sociales. Entre los aspectos que limitan el desarrollo de esta comunidad es la extrema pobreza, ya que carecen de condiciones básicas para sus viviendas, escuelas y lugares de esparcimiento. Las personas trabajan de forma informal en mototaxi, ventas de alimentos, construcción, tiendas y alquiler de video juegos (Departamentos de Policía Huila-DEUIL, 2017). De acuerdo con Gutiérrez (2004), las instituciones educativas ofrecen tres oportunidades a sus estudiantes para que sean físicamente activos, estas son: (1)-los juegos durante el recreo entre las clases, (2)-las clases de educación física que están dentro del currículo, y (3)-las actividades deportivas extracurriculares que se realizan fuera de las instituciones educativas, tal es el caso de la Escuela Juego Limpio, Fútbol, Paz y Convivencia.

\section{El deporte en el proceso formativo}

La educación deportiva como proceso formativo involucra aprendizajes sociales, culturales y psicológicos 
que potencian las destrezas y conectan lo educativo con las realidades de cada estudiante. Por esta razón, se debe pensar en el desarrollo de la mente y el cuerpo, la actividad física y las emociones, la creatividad y la formación en valores que contribuyan a la construcción de la identidad del ser humano (Díaz Monsalve \& Quiroz Posada, 2013). Sin duda, el deporte incorpora valores como la responsabilidad y el trabajo en equipo, a su vez, puede fomentar la integración social y el desarrollo económico en diferentes contextos. Así también puede desarrollar el capital humano y la productividad al mejorar la salud física y mental, y con ello los resultados educativos (Jaitman \& Scartascini, 2017).

El enfoque actual de educación busca que la escuela en conjunto con las familias sean un taller para la vida, al generar procesos de comprensión, respeto y aceptación que posibilite al estudiante incorporar representaciones positivas de las personas con las que interactúa y le permita integrarse adecuadamente en la sociedad (Cantillo, Osorio \& Puello, 2017). En este sentido, las instituciones educativas están llamadas a promover sistemas de aprendizajes integrales, que estimulen al estudiante a aprender, a vivir consigo mismo, a convivir con los demás, a enfrentar las diferentes situaciones y a construir proyectos de vida (Parra, 2003; Pérez, Ramos \& Achón, 2007; Torres, 2012).

En Colombia, uno de esos sistemas de aprendizaje es la educación complementaria, la cual promueve jornadas extracurriculares que se articulan con el currículum. Dichas jornadas tienen como fin ofrecer al estudiante alternativas para un buen uso de su tiempo libre, para lo cual se desarrollan programas que incluyen la recreación, cultura y deporte que complementan las actividades pedagógicas (Ministerio de Educación Nacional de Colombia, 2010). Se espera que estas actividades proporcionen entornos supervisados a los alumnos que prevengan comportamientos antisociales y ofrezcan experiencias enriquecedoras que mejoren sus logros académicos, se fomente la amistad, la cooperación, valores y autoestima (Contreras, 2008; Garrido, Campos \& Castañeda, 2010). Incluso, González, Manrique \& López (2012). sugieren la coordinación entre la actividad física extraescolar y la educación física que se realiza en el centro escolar, lo cual «mejoraría la calidad de ambas actividades, que se complementarían mutuamente» (p.16).

Es conocido que la participación en cualquier deporte favorece el desarrollo de cualidades como lealtad, cooperación, honestidad, tolerancia, dominio de sí mismo, resistencia, respeto, perseverancia, trabajo en equipo, compañerismo, hábitos saludables y construcción de proyectos de vida, lo cual ayuda a entender al deporte por su función en la formación de quienes lo practican (Bravo \& Escobar, 2013; Gutiérrez, 2004). El Fondo de las Naciones Unidas para la Infancia (UNICEF) reconoce que el deporte tiene un papel fundamental en la vida de los niños y niñas, ya que influye en su potencial a nivel físico, emocional, intelectual y social (Gómez, Ahualli \& Pzellinsky, 2013). Para Mireles (2017) algunos de los efectos sociales positivos que producen las actividades deportivas en poblaciones vulnerables de la sociedad son: (1)-la construcción de actitudes y valores y (2)-la construcción de identidad individual y grupal, aspectos que favorecen los procesos de integración social y, por consiguiente, de socialización, lo que permite disminuir las proble- máticas sociales. Rodríguez, Iglesias \& Molina (2020) destacan la relación entre la práctica de actividad física y calidad de vida de los niños, señalan que «altos niveles de actividad física influyen notablemente en la salud general, bienestar físico, autonomía y relación con los padres y entorno escolar» (p.133).

A partir de 1980 algunos países europeos comenzaron a incluir el deporte en su política social como instrumento de lucha contra la exclusión, con el fin de aminorar los efectos de las desigualdades en diferentes poblaciones desfavorecidas (Maza, Balibrea, Camino, Durán, Jiménez \& Santos, 2011). Estos mismos autores han documentado diversas iniciativas deportivas de intervención social. Por ejemplo, L'Agence pour l'Education par le sport en Francia, tiene como finalidad colaborar en el desarrollo de proyectos de inserción y educación a través del deporte, en 2010 puso en marcha el proyecto Experimentación nacional para una política de educación a través del deporte en los barrios populares, con el objetivo de contribuir al desarrollo del deporte educativo en los barrios desfavorecidos. El programa Streetgames en el Reino Unido, es un programa de voluntariado que se inició en 2006 para jóvenes desfavorecidos residentes en barrios con dificultades en el municipio de Reading, ubicado en el condado de Berkshire, en Inglaterra. Este programa desarrolla actividades deportivas en las instalaciones municipales, con el objetivo de promover la integración. En 2007 nace en Francia el programa $L a$ ciudad de Ermont y el deporte ciudadano, con el objetivo de integrar a los vecinos para mejorar su participación en la comunidad a través del voluntariado y desarrollar actitudes de solidaridad. Se organizan diversos campeonatos de fútbol e incluye una política que favorece inserción laboral de los jóvenes del barrio desfavorecido Val-et-Forêt (Maza et al., 2011).

Respecto a estas mismas propuestas en América Latina y el Caribe, Jaitman \& Scartascini (2017) señalan que existe preocupación por la baja actividad física de la población en estas regiones. Desde 2004, el Banco Interamericano de Desarrollo (BID) ha apoyado este tipo de proyectos que se han puesto en marcha en 18 países, entre ellos Colombia. Estas iniciativas se centran en utilizar el deporte para enganchar a jóvenes en programas que incluyen la empleabilidad, las habilidades para la vida, la educación, la salud y el bienestar, la prevención de la violencia, la inclusión social y de género. Sin embargo, existen escasas publicaciones de investigaciones rigurosas que permitan conocer sistemáticamente los efectos de estos programas.

Si bien es cierto que se reconocen los beneficios de la actividad deportiva, Maza et al. (2011) advierten que algunas intervenciones deportivas tendientes a poner en marcha programas de modificación de comportamientos pueden producir efectos negativos, como el rechazo por parte de los jóvenes. Estos mismos autores recomiendan que, para obtener buenos resultados, las instituciones deberían proponer programas que faciliten a los jóvenes una práctica deportiva cercana a sus intereses, al permitirles disfrutarla pues «contribuye a acrecentar el gusto por vivir, la energía personal y el vínculo social» (Maza et al., 2011, p.38). Añaden que también puede resultar valioso incorporar en los proyectos iniciativas de formación laboral y profesional que puedan con- 
tribuir al menos a una mínima independencia económica a medio o largo plazo.

\section{EI fútbol en la educación para la vida}

El fútbol es un deporte que favorece el trabajo en equipo y las habilidades sociales, «a la vez promueve un estilo de vida saludable ya que mejora las cualidades motrices y las capacidades físicas condicionales en aquellas personas que practican este deporte» (Barragán, Cepeda \& Ferro, 2016, p.43). Además de obtener mejoras a nivel motor y de condición física, el fútbol puede tener una incidencia directa en el rendimiento académico (Ordóñez, Polo, Lorenzo \& Shaoliang, 2019). El fútbol puede ser considerado como un deporte popular que busca una forma de comunicación en y con el mundo ya que une a todos bajo un mismo objetivo, sin importar las razas, edad o estatus social (González \& Ramírez, 2014; Gómez et al., 2013). Así también, dota de identidad a quien no encuentra grupo de referencia de manera natural, ya que da sentido de pertenencia (Valderrama, SolisEspallargas, Trigueros, Manjón \& Limón, 2015). De igual modo, se educa en la tolerancia y dominio de sí mismo, responsabilidad, a aceptar tanto la victoria como el fracaso y a respetar al adversario (Garrido et al., 2010). El fútbol como toda actividad deportiva, cuando el enfoque no es educativo, puede tener desventajas como la exclusión de los alumnos menos competentes, situaciones que se deben cuidar, en opinión de Maza et al. (2011) mediante la revisión y replanteamiento de los objetivos de la intervención social y educativa, así como del perfil de los entrenadores y educadores.

Una de las iniciativas exitosas, que tiene por objetivo la promoción de la integración y el desarrollo a través del fútbol, es Street football world, organización alemana no gubernamental. Para esta organización, la práctica del fútbol es una herramienta de cambio que puede utilizarse para promover la educación, la integración, la salud y la paz. Cuenta con más de 80 iniciativas deportivas para la integración social, que permiten a sus participantes compartir las mejores prácticas, crear nuevas asociaciones y enriquecer sus propios programas basándose en las experiencias de los demás (Maza etal., 2011).

Escamilla, Alguacil \& González-Serrano (2020) indican que la motivación es el factor más importante para lograr que los alumnos mantengan la práctica deportiva fuera del ámbito escolar. Fraile, Tejero-González, Esteban-Cornejo \& Veiga (2019) coinciden en señalar que los alumnos con bajo disfrute, baja autoeficacia motriz y bajo nivel de frecuencia de práctica, tienen mayor posibilidad de abandonar las prácticas deportivas. Martinek, Holland \& Seo (2019) señalan que la motivación de los alumnos para sostener la actividad física parece estar relacionada con sus creencias y valores acerca del programa y del entrenador deportivo. Al respecto, Almonacid-Fierro, Merellano-Navarro, Feu, Vizuete \& Orellana (2019), indican que los profesores-entrenadores requieren conocimiento didáctico que les permita planificar y poner en práctica estrategias de enseñanza-aprendizaje con el fin de alcanzar los objetivos para la formación de los estudiantes. Además, Baños \& Arrayales (2020) destacan la importancia de planificar las prácticas deportivas con enfoque en la satisfacción por el aprendizaje, «ya que se puede tener una gran repercusión en el aumento de la actividad física en el tiempo libre de los adolescentes, puesto que se potenciaría la satisfacción, diversión y la motivación intrínseca» (p.86). En este sentido, el interés por sostener la actividad física, es influido por las disposiciones iniciales individuales, las relaciones programa deportivo-entrenador-alumno, y la motivación.

Cantillo et al. (2017) destacan que las interacciones positivas entre los agentes educativos ofrecen a los alumnos una imagen de acercamiento entre las personas con las que interactúan, lo que favorece la socialización y la autonomía personal. Para ello, se sugiere que el entrenador posea habilidades sociales, conocimientos básicos sobre dinámicas de grupo y resolución de conflictos, así como tener espacios de comunicación entre profesores y entrenadores que participan en el proyecto deportivo, con el fin de compartir información sobre situaciones del grupo de alumnos y la clarificación de las funciones. El objetivo de estos espacios de comunicación es lograr la comprensión y reflexión sobre situaciones del grupo (Maza et al., 2011).

Con estos antecedentes, este estudio de tipo exploratorio se propuso comprender desde la perspectiva de los participantes la manera en la que la Escuela de Fútbol Juego Limpio contribuye en la formación de los alumnos.

\section{Método}

Dado que el objetivo de esta indagación es comprender la manera en la que la Escuela contribuye en la formación de los alumnos, el estudio fue conducido con enfoque cualitativo, orientación que permitió recoger datos desde las perspectivas y vivencias de los participantes (Merriam, 2015).

\section{Participantes}

A fecha de este estudio la Escuela contaba con 60 alumnos inscritos, de entre cinco y 15 años, los cuales tenían como requisito para pertenecer a la misma, estar escolarizados en algún centro educativo en primaria o secundaria. Además, en la Escuela se cuenta con la participación de docentes de apoyo académico y de los padres de familia como acompañantes en el proceso formativo-deportivo.

Se seleccionaron 20 participantes distribuidos de la siguiente manera: seis niños y cuatro niñas pertenecientes a las categorías propias de la Escuela con el nombre de pony (8 a 10 años), pre-infantil (11 y 12 años) e infantil (13 a 15 años) inscritos en la Escuela, cinco madres de alumnos y

\begin{tabular}{|c|c|c|c|c|c|}
\hline \multirow{2}{*}{$\frac{\text { No.Código identificación }}{1 \text { J.K.S.M }}$} & \multirow{2}{*}{$\begin{array}{l}\text { Género } \\
\text { Femenino }\end{array}$} & \multirow{2}{*}{$\begin{array}{l}\text { Edad } \\
9\end{array}$} & \multirow{2}{*}{$\begin{array}{l}\text { Categoría } \\
\text { Pony }\end{array}$} & \multicolumn{2}{|c|}{ Tipo de participante Grado educativo } \\
\hline & & & & Estudiante & $4^{\circ}$ \\
\hline 2 A.F.T.C. & Masculino & 8 & Pony & Estudiante & $4^{\circ}$ \\
\hline 3 M.N.V & Femenino & 13 & Infantil & Estudiante & $8^{\circ}$ \\
\hline 4 S.F.A.O & Masculino & 10 & Pony & Estudiante & $5^{\circ}$ \\
\hline 5 K.L.C.R. & Masculino & 12 & Pre-infantil & Estudiante & $7^{\circ}$ \\
\hline 6 J.D.C.LL. & Masculino & 15 & Infantil & Estudiante & $11^{\circ}$ \\
\hline 7 J.E.M.C & Masculino & 14 & Infantil & Estudiante & $9^{\circ}$ \\
\hline 8 L.V.CH.M. & Femenino & 11 & Pre-infantil & Estudiante & $6^{\circ}$ \\
\hline 9 I.C.R. & Masculino & 9 & Pony & Estudiante & $4^{\circ}$ \\
\hline 10 J.K.P. & Femenino & 13 & Infantil & Estudiante & $9^{\circ}$ \\
\hline 11 M.LL.R & Femenino & 45 & N.A. & Madre de familia & $8^{\circ}$ \\
\hline 12 Y.P.A. & Femenino & 48 & N.A. & Madre de familia & $7^{\circ}$ \\
\hline 13 Y.P.M.G & Femenino & 43 & N.A. & Madre de familia & $4^{\circ}$ \\
\hline 14 E.R.Q & Femenino & 36 & N.A. & Madre de familia & $10^{\circ}$ \\
\hline 15 C.Q. L. & Femenino & 27 & N.A. & Madre de familia & $11^{\circ}$ \\
\hline 16 Z.C.C. & Femenino & 36 & N.A. & Docente & Universitario \\
\hline 17 D.M.D. & Femenino & 33 & N.A. & Docente & Universitario \\
\hline 18 L.J.P. & Femenino & 30 & N.A. & Docente & Universitario \\
\hline 19 E.S.S. & Femenino & 32 & N.A. & Docente & Universitario \\
\hline 20 F.H.C. & Femenino & 31 & N.A. & Docente & Universitario \\
\hline
\end{tabular}


cinco docentes de apoyo académico que participaban de manera voluntaria en la Escuela. Todos firmaron cartas de consentimiento informado. La elección de los participantes se realizó de manera intencionada debido a su accesibilidad para participar en la investigación, y proporcionar datos en relación al objetivo de la misma (Izcara, 2014 y Merriam, 2015). Con la finalidad de cuidar la confidencialidad de los participantes, se siguió la imposición de anonimato sugerido por Gibbs (2014), de ese modo se omitieron los nombres reales y se asignaron códigos para su identificación (véase tabla 1).

\section{Técnicas para la recogida de datos}

Los datos fueron obtenidos mediante observación participante, entrevistas semiestructuradas individuales y grupos focales (Merriam, 2015). Se realizaron cuatro observaciones de 50 minutos cada una en las instalaciones deportivas, se observó a todos los participantes durante las actividades deportivo-académicas. Para el registro de los datos de observación se utilizó el diario de campo. En un primer momento, se diseñaron y aplicaron las entrevistas de manera individual a los alumnos, a las madres de alumnos y a las docentes de apoyo académico. Posteriormente, con la intención de profundizar en algunos temas derivados de las observaciones y entrevistas individuales, se diseñaron las entrevistas grupales. Se llevaron a cabo dos grupos focales, uno con las docentes y otro con las madres.

\section{Proceso analítico}

Para el análisis de los datos se siguió la propuesta de Izcara (2014) y Merriam (2015), la cual consiste en: (1)-la simplificación de la información; (2)-la categorización de la información. En el primer paso se realizó la reducción de los datos analizando su importancia o pertinencia, y en el segundo paso se llevó a cabo un ordenamiento de temas por categorías o por bloques temáticos.

El proceso de validación y triangulación consistió en mostrar como un determinado resultado obtenido por medio de la entrevista coincide o no con el resultado obtenido por observaciones, o en la entrevista con otra persona, para así dar apoyo o reforzar dicho resultado (Lincoln \& Guba, 1985; en Valenzuela \& Flores, 2012). En relación a la validez en los estudios cualitativos se ha tenido en cuenta el enfoque de Taylor \& Bogdan (2006) que recomiendan el ajuste entre el análisis y los datos. Por ello, en la presentación de los resultados se incluyen comentarios textuales de los participantes.

\section{Resultados}

Los resultados se organizaron en dos categorías: (1)promoción del crecimiento personal y académico; y (2)-transformación y proyecto de vida. En la tabla 2 se esquematizan dichas categorías y su significado, posteriormente se describen los resultados de cada una.

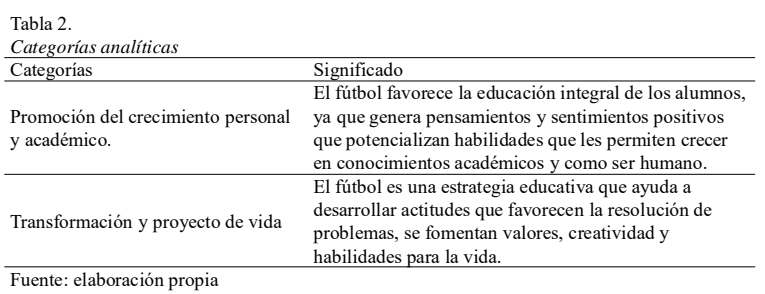

\section{Promoción del crecimiento personal y académico}

En esta categoría se describen los resultados acerca de la participación que ha tenido la Escuela en la formación de los alumnos, tanto en el ámbito deportivo como en el académico.,

De forma coordinada, junto a las prácticas de fútbol y actividades lúdico-pedagógicas se llevan a cabo asesorías para el reforzamiento de las asignaturas como lectura y matemáticas. En la tabla 3 se presentan los resultados encontrados en esta categoría.

\begin{tabular}{|c|c|c|c|}
\hline \multirow[b]{2}{*}{ Categoría } & \multicolumn{3}{|l|}{ Resultados } \\
\hline & Alumnos & Docentes & Madres de familia \\
\hline $\begin{array}{l}\text { Promoción del } \\
\text { crecimiento personal y } \\
\text { académico }\end{array}$ & $\begin{array}{l}\text { Apoya en el desempeño } \\
\text { escolar. } \\
\text { Ayuda a ser responsable, } \\
\text { dedicado. } \\
\text { Ayuda a tener un mejor } \\
\text { comportamiento. } \\
\text { Conseguir amigos. }\end{array}$ & $\begin{array}{l}\text { Actividades } \\
\text { lúdico/pedagógicas que } \\
\text { refuerzan la formación } \\
\text { integral. } \\
\text { Se convierte en un } \\
\text { agente de paz para ellos } \\
\text { y sus familias. } \\
\text { Se implementan normas } \\
\text { y valores. } \\
\text { Se desarrolla la } \\
\text { capacidad de resolver } \\
\text { conflictos. }\end{array}$ & $\begin{array}{l}\text { Busca la formación } \\
\text { integral. } \\
\text { Evita que los niños } \\
\text { y niñas caigan en } \\
\text { vicios. } \\
\text { Los niños aprenden } \\
\text { a cumplir reglas y } \\
\text { normas. } \\
\text { Fortalecen los } \\
\text { valores y a } \\
\text { solucionar los } \\
\text { conflictos que se } \\
\text { les presentan en la } \\
\text { vida diaria. }\end{array}$ \\
\hline
\end{tabular}

El 100\% de los participantes señalaron que la Escuela ayuda a los alumnos en su formación, debido a las actividades deportivas y asesorías académicas que se llevan a cabo, respecto a éstas últimas se refuerza la lectura a través de cuentos e historias, se realizan ejercicios y solución de problemas matemáticos, dibujos y obras de teatro, así como actividades para el fomento de hábitos de higiene y vida saludable. Para los alumnos, practicar fútbol contribuye a la educación académica porque se adquieren hábitos de estudio, disciplina y responsabilidad. Algunos de los comentarios de los niños y adolescentes entrevistados fueron: «aporta conocimientos» (JCSM), «ayuda a tener disciplina, responsabilidad, uso del tiempo libre» (JDCLL), «ayuda a ser responsable, dedicado, a tener un mejor desempeño en el colegio» (JSMC), «ayuda para ser organizado con mis cuadernos» (SFAO), «el cambiar de ambiente ayuda a la formación académica» (KLCR).

Todos los alumnos (100\%), coinciden en señalar que el asistir a la Escuela, y más concretamente, practicar fútbol, les ha ayudado en su vida personal. Algunas de las apreciaciones realizadas por ellos han sido comentarios tales como «me ha ayudado a mejorar en el comportamiento» (LVCHM), «tener un mejor comportamiento, comparto con más niños y niñas» (JCPM), «al jugar fútbol, se aprenden valores y comportamientos» (JCSM) «distrae y ayuda a pensar en algo diferente para asumir las tareas» (JCPM). De igual forma manifestaron que dentro de sus prácticas deportivas se han creado lazos de amistad, como ejemplo uno de los niños entrevistados expresó «he conseguido amigos, disfruto de cada actividad» (AFTC).

Todas las docentes $(100 \%)$, comentaron que el fútbol ayuda a los niños a tener disciplina y favorecer la formación integral, una de ellas dijo: «Tiene como objetivo recrear a los niños, fomentar disciplina y forjar para un futuro» (FEC), otra de las docentes entrevistadas comentó: «El futbol para los niños y niñas es muy importante porque además de implementar un deporte, busca la formación integral en las diferentes áreas» (ESS). De acuerdo con las opiniones de las 
docentes, el fútbol ayuda a que los alumnos tengan una motivación para sus vidas, lo cual se ve reflejado en el aprendizaje y comportamientos, pero especialmente se ve reflejado en su motivación, y a este respecto una docente declaró «el futbol es para los niños del asentamiento algo que les agrada, una de las más grandes motivaciones, es una herramienta importante para formarlos en todos los aspectos» (ZC).

Para el 100\% de las docentes, con la práctica del fútbol se fortalecen los valores como el respeto, la responsabilidad y trabajo en equipo, y se adquieren normas de comportamiento y resolución de conflictos mediante el diálogo que se establece durante las asesorías académicas y la práctica deportiva. Una de las docentes entrevistadas comentó «el fútbol, como una disciplina que se practica con constancia, favorece la responsabilidad y adquisición de normas de comportamiento que pueden conducir a un cambio social en las nuevas generaciones» (LJP). Otra docente dijo «la educación escolar y la práctica del deporte se complementan, ya que por medio de éste los niños aprenden a cumplir reglas, también a fortalecer los valores y aprenden a solucionar los conflictos que se les presentan en la vida diaria» (FEC).

Todas las madres (100\%), enfatizaron la importancia de la Escuela en cuanto al aprovechamiento del tiempo libre de los alumnos y el tener mejores relaciones sociales. Una de las madres señaló «el buen uso y aprovechamiento del tiempo libre evita que los niños caigan en vicios o que estén haciendo travesuras a la comunidad; es importante porque aprenden valores y piensan en otras cosas» (MLLR). Otra madre comentó «el deporte hace que se ocupe el tiempo libre de los niños y niñas y alejarlos de las problemáticas sociales» (GPM). El aprovechamiento del tiempo libre de los niños y niñas en actividades deportivas, tiene aún más relevancia en este contexto, tomando en cuenta que el sector es vulnerable y se presentan dificultades de tipo social relacionadas con la drogadicción y pandillerismo. De igual modo, se justificó el potencial que posee el futbol y su práctica para el bienestar académico, personal, familiar y de la comunidad. En este sentido, tres madres de los alumnos (60\%), dijeron que se puede convertir en un agente de paz y convivencia «con las actividades deportivas se comparte en familia y entre amigos» (ERQ), «el practicar un deporte los previene de malas compañías o malas amistades» (KC), «la Escuela permite a los niños y niñas realizar actividades que les ayudan a formarse como personas y estudiantes» (GPM).

De acuerdo con los resultados, la práctica de fútbol y las actividades pedagógicas desarrolladas en la Escuela, son estrategias educativas valiosas que han permitido que los alumnos desarrollen habilidades motrices, cognitivas y sociales. Por tanto, la Escuela se ha convertido en un espacio de recreación, aprendizaje, convivencia y apertura para manejar situaciones problemáticas. Los participantes resaltan la importancia de esta iniciativa en el buen aprovechamiento del tiempo libre por parte de los alumnos, especialmente si se tienen en cuenta las características socio-económicas de la comunidad a la que pertenecen.

\section{Transformación y proyecto de vida}

En esta categoría se describen los resultados acerca del cambio en el estilo de vida que han tenido los alumnos al participar en la Escuela, así como la manera en la que se han desarrollado habilidades creativas, estilos de vida saludables y construcción de proyectos de vida. En la tabla 4 se presentan los resultados encontrados en esta categoría.

\begin{tabular}{|c|c|c|c|}
\hline \multirow{2}{*}{ Categoría } & \multicolumn{3}{|l|}{ Resultados } \\
\hline & Alumnos & Docentes & Madres de familia \\
\hline $\begin{array}{l}\text { Transformación } \\
\text { y proyecto de } \\
\text { vida }\end{array}$ & $\begin{array}{l}\text { Antes veía televisión, dormir, } \\
\text { hacer la tarea, jugar en la } \\
\text { calle. } \\
\text { Ahora en la escuela jugar } \\
\text { fútbol, reforzar temas del } \\
\text { colegio, se aprenden valores y } \\
\text { a ser más tolerantes. } \\
\text { Cuando crezca voy a ser un } \\
\text { futbolista profesional, } \\
\text { profesor o entrenador físico. }\end{array}$ & $\begin{array}{l}\text { Ambiente sano y } \\
\text { promoción de proyecto } \\
\text { de vida. } \\
\text { Se desarrollan } \\
\text { habilidades creativas. } \\
\text { Son niños y niñas con } \\
\text { sueños que desean } \\
\text { aprender. } \\
\text { Cambia sus estilos de } \\
\text { vida y practican un } \\
\text { deporte que les apasiona. }\end{array}$ & $\begin{array}{l}\text { Medio para } \\
\text { transformar vidas, } \\
\text { familias y sociedad. } \\
\text { Da disciplina y una } \\
\text { mirada diferente de la } \\
\text { vida. } \\
\text { Con orientación se } \\
\text { puede transformar la } \\
\text { realidad al crear } \\
\text { proyectos de vida. }\end{array}$ \\
\hline
\end{tabular}

Los alumnos (100\%), manifestaron haber experimentado cambios en sus actividades de tiempo libre, antes y después de pertenecer a la Escuela de fútbol. Uno de los alumnos que realizaron el cuestionario (JCPM) reconoció que antes de pertenecer a la Escuela de fútbol veía la televisión, jugaba, hacia tareas, y ahora además de jugar al fútbol, reforzaba tareas y actividades pedagógicas. Otras de las descripciones antes-ahora por parte de alumnos de la Escuela, han sido: «antes veía televisión, hacer tareas, dormir. Ahora estudio en la Escuela de futbol y veo menos televisión» (JDCLL), «me iba para la cancha a ver jugar y me la pasaba por fuera de la casa, ahora en la Escuela me siento feliz porque juego y me dan refrigerios» (AFTC).

Las manifestaciones por parte de los participantes en el estudio, revela el cambio positivo que ha tenido lugar en los alumnos al asistir a la Escuela de fútbol. Cabe señalar que la Escuela ha orientado a los alumnos a pensar en un proyecto de vida que les permita ver más allá de su realidad. Los alumnos en su gran mayoría (80\%), desean dedicarse a una disciplina en la línea del deporte, como es el caso del fútbol, ya sea como futbolista profesional, árbitro o educador físico. En este sentido algunas de sus respuestas en la entrevista fueron, entre otras: «me gustaría ser futbolista, pero también ser profesora» (JCPM); «quisiera ser futbolista y educador físico, porque me apasiona y lo hago a diario» (JDCLL).

Para todos los alumnos, el aprender a jugar y el estar en un espacio donde se pueden expresar libremente, les ha permitido ser tolerantes, lo cual enriquece su aprendizaje. Algunos de los muchachos que contestaron la entrevista manifestaron entre otras ideas: «es una actividad divertida que complementa la vida social y personal, hemos aprendido valores y a ser más tolerantes» (JCSM). En la Escuela, los alumnos han tenido una formación humana que les ha servido en su vida cotidiana. Dos participantes respondieron textualmente: «aprendo valores, a hacer tareas, a ser más dedicada» (MVM), «es importante porque me da bases necesarias para aplicar los valores» (LVCHM).

El 100\% de las docentes expresaron que el pertenecer a la Escuela ayuda a los alumnos a estar en un ambiente sano, a crear un proyecto de vida y con ello transformarla. A modo ilustrativo una docente dijo: el fútbol ayuda a que en sus tiempos libres compartan con los demás en un ambiente sano y ayuda a la formación de su proyecto de vida (FEC). Otra docente expresó textualmente: «dentro del contexto hay 
muchos niños con habilidades para este deporte, lo cual a largo plazo puede ser exitoso y productivo para ellos» (ZC).

Asimismo, en opinión del 100\% de las docentes, los alumnos desean cambiar sus estilos de vida y tal vez tener la oportunidad de demostrar sus destrezas y habilidades. A este respecto, dos docentes comentaron textualmente: «se puede entender que quienes asisten a la Escuela de fútbol, son los niños y niñas con sueños que desean aprender y practicar un deporte que les apasiona, en este caso el fútbol» (DMD), «los niños y niñas demuestran interés en participar de las actividades deportivas, por ello asisten puntualmente» (ESS). El 100\% de las docentes señalaron que con la práctica del deporte se desarrollan habilidades creativas para el dibujo, la pintura, la actuación y manualidades en las que se elaboran objetos con diferentes materiales. Dos de las docentes entrevistadas, nos ilustran esta idea y textualmente reconocieron: «se observan cualidades en algunos alumnos en actuación, en lectura, habilidades para las matemáticas y de memorización» (FHC), otra docente dijo: «habilidades y destrezas creativas que en ocasiones no son potencializadas en la Escuela» (FEC).

Por su parte, todas las madres de los alumnos (100\%), resaltaron la orientación que brinda el deporte en la construcción de proyectos de vida. Algunas de las apreciaciones por parte de estas madres fueron textualmente: «el futbol además de ser divertido puede llegar a ser un medio para el crecimiento profesional «(ER), otros comentarios al respecto «a los niños y niñas que les gusta, el fútbol les da disciplina y una mirada de la vida diferente» (GPM), «un niño que desea ser futbolista puede llegar a serlo con una persona que lo oriente, entrene y le enseñe a ser una buena persona para la sociedad» (DMC) y «los proyectos de deporte para nuestros hijos sirven para sacarlos adelante con el apoyo de la universidad» (ERQ).

Los resultados sugieren que, en la Escuela, la práctica de fútbol facilita la recreación y el intercambio social. La disciplina y la forma de abordarla con los niños y jóvenes, ha llegado a ser reconocida como valor para su desarrollo y bienestar, permitiéndoles cambiar sus comportamientos y ayudándoles a construir un proyecto de vida que les genere mejorar sus condiciones de vida.

\section{Discusión}

Este estudio se propuso averiguar la manera en la que la Escuela de Fútbol Juego Limpio contribuye en la formación de los alumnos. Dentro de los principales hallazgos se encontró que practicar fútbol promueve estilos de vida saludable, mejora las habilidades motrices y cognitivas lo cual coincide con los trabajos de Barragán et al. (2016), Gutiérrez (2004), Jaitman \& Scartascini (2017). Así también, hemos comprobado que las actividades desarrolladas en la Escuela influyen en el aprovechamiento académico. De acuerdo con Ordóñez et al. (2019) el incremento en el rendimiento académico como resultado de la actividad física parece estar relacionado con el aumento en los niveles atencionales y la mejora en el procesamiento de la información.

Los resultados sugieren que la práctica del fútbol es un proceso de aprendizaje que permite a los alumnos adquirir cualidades como la disciplina, tolerancia, respeto, generar lazos de amistad y compañerismo. Todos estos valores, favorecerían una mayor inclusión social, participación comunitaria y disminuir las problemáticas sociales que se presentan en las comunidades (Bravo \& Escolar, 2013; Murillo, 2017).

La Escuela de fútbol se ha convertido en un espacio de entretenimiento y aprendizaje que estimula en los alumnos hábitos alimenticios, de higiene y de comportamiento saludables y a tener sueños e ilusiones encaminados a crear proyectos de vida orientados al estudio y al deporte, forjando así, el establecimiento de metas que conducen al crecimiento personal con el fin de mejorar sus condiciones de vida. Esto amplía la comprensión acerca de que el deporte no es únicamente una forma de ejercitar el cuerpo, sino que también influye en la formación de los alumnos que lo practican, lo cual puede derivan en una mejor calidad de vida (Bravo \& Escobar, 2013; Gutiérrez, 2004; Rodríguez et al., 2020).

Adicional al desarrollo de habilidades motrices, cognitivas, y sociales, se encontró que la práctica del fútbol estimula la creatividad para el dibujo, la pintura y la actuación. Lo anterior sugiere que el deporte, y en particular el fútbol, contribuye a la estimulación de habilidades para el desarrollo del talento de los alumnos. De ahí la importancia de continuar con esta iniciativa en coordinación con las familias, ya que ambas actúan como agentes socializadores. Cantillo et al. (2017), De León (2011), Maza et al. (2011) destacan la importancia del trabajo conjunto de ambas instituciones en la enseñanza de forma integral.

Cabe destacar que, durante el desarrollo de la investigación, se observó que, para las madres de familia, la Escuela ha significado un lugar en el cual sus hijos pueden desarrollarse con disciplina, mejorar la socialización y aprovechamiento escolar. También se observó, por parte de las madres, un compromiso por colaborar en la mejora de las condiciones de las infraestructuras. Entre otras mejoras, colaboraron en la colocación de las mallas exteriores para la detención de los balones puesto que el campo de fútbol está cerca de un afluente de río. Otra colaboración que realizaron las madres de los alumnos de la Escuela, fue la construcción de una pequeña biblioteca con libros donados por la comunidad. Esto permitió tener un espacio para investigar y realizar sus tareas junto a las docentes, ya que, el horario de estudio en las instituciones educativas y el tiempo para realizar las tareas en casa, provocaba la falta de tiempo para entrenar.

El trabajo del entrenador físico y de las siete docentes de apoyo psicopedagógico es valorado en forma significativa por los alumnos y madres de familia, ya que el desarrollo de la práctica del fútbol en conjunto con actividades académicas y creativas fortalecen los aprendizajes y las interacciones entre los participantes. Estos resultados sugieren que existe un acercamiento respetuoso y de compañerismo entre los educadores, alumnos y familias, lo que posiblemente contribuya al sostenimiento de la motivación de los alumnos en cuanto a la asistencia y pertenencia a la Escuela. Esto coincide con lo expuesto por Almonacid-Fierro et al. (2019), Escamilla et al. (2020), Baños \& Arrayales (2020), Fraile et al. (2019), Martín-Barrero \& Martínez-Cabrera (2019) \& Martinek et al. (2019) en cuanto a la relación entre la motivación y autoeficacia en la realización de actividades deportivas con la calidad de la planeación, los recursos, las estrategias de 
enseñanza-aprendizaje y los vínculos con los profesoresentrenadores.

\section{Conclusiones}

Este estudio destaca que la práctica de fútbol con acompañamiento académico promueve el crecimiento personal de los alumnos que participan en la Escuela Juego Limpio, Fútbol, Paz y Convivencia, al promover el desarrollo de pensamientos, sentimientos, y potencializar habilidades motoras, cognitivas y sociales que les permiten crecer deportivamente y en conocimientos académicos. Así también, respecto a su aportación a la transformación y generación de proyectos de vida, el fútbol contribuye como estrategia deportivoeducativa al facilitar el desarrollo de actitudes para resolver problemas, fomentar valores, creatividad y compañerismo.

Sin duda, los beneficios de la práctica de fútbol y el acompañamiento educativo que reciben los alumnos en esta Escuela, es el resultado de la participación conjunta y compromiso del entrenador físico, docentes, alumnos y familias, quienes a pesar de las condiciones económicas y de infraestructuras poco favorables en las que se desarrolla esta iniciativa, participan cada uno desde su propio rol, teniendo un objetivo común: el desarrollo físico, social y académico de los alumnos. Todos ellos se inspiran en el afán de aprender y crecer, y en la imagen de un proyecto que en un futuro pudiese mejorar las condiciones de vida y del entorno de estos alumnos y sus familias.

Realizar un estudio longitudinal en el cual se analicen las trayectorias personales, académicas, laborales y profesionales de los alumnos titulados, pudiera aportar conocimiento valioso de los efectos en el desarrollo y logros a medio y largo plazo derivados de su pertenencia a esta Escuela. Se recomienda también realizar una futura investigación, que se proponga y analice el trabajo articulado entre la Escuela y las familias, con el propósito de generar procesos comunitarios de aprendizaje que se comprometan en la construcción de proyectos para la mejora de las condiciones de vida de la comunidad. Otra línea de indagación pudiese ser el ampliar esta iniciativa a otras comunidades poco favorecidas, de tal modo que sus habitantes puedan conocer y beneficiarse a este tipo de experiencias deportivas con acompañamiento académico.

Así también, es recomendable fortalecer el equipo de trabajo en la Escuela, específicamente en la contratación de al menos dos entrenadores y un técnico deportivo, tres graduados en educación infantil, un auxiliar de enfermería y un asistente de servicios generales. Todo ello permitirá, con mayor facilidad, alcanzar los objetivos deportivos/formativos que permitan a los alumnos ascender en el plano deportivo, esto es, jugar profesionalmente en las selecciones pre-juveniles y juveniles.

Proponemos impulsar políticas gubernamentales que apoyen proyectos de desarrollo social mediante el deporte. Esto es especialmente urgente en países como Colombia y más concretamente en las zonas con mayor desigualdad y con menor porcentaje de escolarización. Estos proyectos pueden contribuir al desarrollo de las capacidades físicas, intelectuales sociales y artísticas de infantes y jóvenes de estas áreas vulnerables o poco favorecidas, para beneficio personal, familiar y de toda la comunidad.

\section{Referencias}

Alcaldía de Garzón-Huila. (2016). Sitio oficial de Garzón en Huila, Colombia. Recuperado de http://www.garzonhuila.gov.co/

Almonacid-Fierro, A., Merellano-Navarro, E., Feu Molina, S., Vizuete Carrizosa, M., \& Orellana Fernández, R. (2019). Perspectiva cualitativa en la construcción del conocimiento didáctico del contenido del profesorado de Educación Física (Qualitative perspective in the construction of didactic content knowledge in Physical Education teachers). Retos, 36, 459-468. Recuperado de https:// recyt.fecyt.es/index.php/retos/article/view/68840/43948

Baños, R., \& Arrayales, E. (2020). Predicción del aburrimiento en la educación física a partir del clima motivacional (Prediction of boredom in physical education from the motivational climate). Retos, 38, 83-88. Recuperado de https://recyt.fecyt.es/index.php/retos/article/view/74301/ 47068

Barragán, S. C., Cepeda, B. J. \&Ferro, B. J. (2016). Propuesta para el desarrollo de la competencia ciudadana de convivencia y paz, en adolescentes de 12 y 13 años del Club Deportivo C2, Sede barrio República de Canadá (Tesis de grado, Corporación Universitaria Minuto de Dios, Colombia). Recuperado de https:// repository.uniminuto.edu/bitstream/handle/10656/4470/ TEFIS_CepedaBarbosaJairoAndres_2016.pdf? sequence $=1$ Bravo, C. N., \& Escobar, R S. (2013). El deporte como medio de inclusión en condiciones de vulnerabilidad (Tesis de grado, Universidad del Valle, Calí, Colombia). Recuperado de http://bibliotecadigital.univalle.edu.co/bitstream/ 10893/4366/1/CB-0472476.pdf

Cantillo, P. J., Osorio, G. A. \& Puello, P. Y. (2017). Relación familia-escuela en la formación integral del niño de la Institución Educativa Ana María Vélez de Trujillo sede acción comunal San Pedro y libertad de Cartagena de indias (Tesis de grado, Universidad de Cartagena, Colombia). Recuperado de http:// repositorio.unicartagena.edu.co/handle/11227/5149

Contreras, J. O. (2008). El deporte escolar como generador de ambientes de aprendizaje excelentes. Revista Fuentes, 8, 8-20. Recuperado de https:// revistascientificas.us.es/index.php/fuentes/article/view/ 2453/2321

De León, S. B. (2011). La relación familia-escuela y su repercusión en la autonomía y responsabilidad de los niños/as. XII Congreso Internacional de Teoría de la Educación por la Universidad de Barcelona, España. Recuperado de http://extension.uned.es/ archivos_publicos/webex_actividades/4440/ larelacionfamiliaescuelaysurepercusionenlaautonomiay.pdf Departamento de Policía Huila-DEUIL. (2017). Informe: fenómenos de orden público. Recuperado de https:// www.policia.gov.co/huila

Díaz Monsalve, A. E., \& Quiroz Posada, R. E. (2013). La formación integral: Una aproximación desde la investigación. Íkala, revista de lenguaje y cultura, 18(3), 17-29. Recuperado de http://www.scielo.org.co/pdf/ikala/v18n3/ 
v18n3a2.pdf

Escamilla Fajardo, P., Alguacil Jiménez, M., \& González-Serrano, M. (2020). Variables predictoras de la motivación deportiva en estudiantes de secundaria federados y no federados (Variables predicting sports motivation in federated and non-federated high school students). Retos, 38, 58-65. Recuperado de https://recyt.fecyt.es/ index.php/retos/article/view/73551

Fraile García, J., Tejero-González, C., Esteban-Cornejo, I., \& Veiga, Ó. (2019). Asociación entre disfrute, autoeficacia motriz, actividad física y rendimiento académico en educación física (Association between enjoyment, motor selfefficacy, physical activity and academic performance in physical education). Retos, 36 58-63. Recuperado de https://recyt.fecyt.es/index.php/retos/article/view/63035

Garrido, G. M., Campos, M. M del C., \& Castañeda, V. C. (2010). Importancia de los padres y madres en la competición deportiva de sus hijos. Revista Fuentes, 10, 173194. Recuperado de https://revistascientificas.us.es/ index.php/fuentes/article/view/2595

Gibbs, G. (2014). El análisis de datos cualitativos en investigación cualitativa. Madrid, España: Morata.

Gómez, A., Ahualli, M., \&Pzellinsky, R. (2013). Si jugamos juntos, ganamos todos. Recomendaciones para entrenamientos de fútbol de niños, niñas y adolescentes. Informe de investigación Fondo de las Naciones Unidas para la Infancia, Argentina. Recuperado de https:// www.unicef.org/argentina/informes/si-jugamos-juntosganamos-todos

González, E. C., \& Ramírez, P. C. (2014). Caracterización de la velocidad de desplazamiento en los futbolistas de la categoría sub-17 participantes de la liga de futbol de Bogotá (Tesis de grado). Corporación Universitaria Minuto de Dios, Bogotá, Colombia. Recuperado de https:/ /repository.uniminuto.edu/bitstream/handle/10656/3350/ TEFIS_GonzalezEspinosaCindy_2014.pdf?sequence=1\&isAllowed=y

González Pascual, M., Manrique Arribas, J., \& López Pastor, V. (2012). Valoración del primer curso de implantación de un programa municipal integral de deporte escolar. (Evaluation of a Integral School-Sports Program first year development). Retos, 21, 14-18. Recuperado de https:// recyt.fecyt.es/index.php/retos/article/view/34597

Gutiérrez, S. M. (2004). El valor del deporte en la educación integral del ser humano. Revista de educación, 335, 105 126. Recuperado de http:// www.revistaeducacion.educacion.es/re335/re335_10.pdf

Izcara, P. S. (2014). Manual de investigación cualitativa. México: Fontamara.

Jaitman, L., \& Scartascini, C. (2017). Deporte para el desarrollo. Informe Banco Interamericano para el Desarrollo.

Recuperado de https://publications.iadb.org/ publications/spanish/document/Deporte-para-eldesarrollo.pdf

Martín-Barrero,A., \& Martínez-Cabrera, F. (2019). El modelo de juego en el fútbol. De la concepción teórica al diseño práctico (Game models in soccer. From theoretical conception to practical design). Retos, 36, 543-551. Recuperado de https://recyt.fecyt.es/index.php/retos/ article/view/71021/43960

Martinek, T., Holland, B., \& Seo, G. (2019). Understanding
Physical Activity Engagement in Students: Skills, Values, and Hope. RICYDE. Revista internacional de ciencias del deporte, 55(15), 88-101. https://doi.org/10.5232/ ricyde2019.05506

Maza, G. (Coord.). Balibrea, K., Camino, X., Durán, J., Jiménez, P., \& Santos, A. (2011). Deporte, actividad física e inclusión social. Una guía para la intervención social a través de las actividades deportivas. Madrid: Consejo Superior de Deportes. Recuperado de http:// www.planamasd.es/sites/default/files/programas/medidas/actuaciones/WEB-GUIA+I.pdf

Merriam, S. (2015). Qualitative research. A guide to design and implementation. San Francisco, CA: Jossey Bass.

Ministerio de Educación Nacional de Colombia. (2010). Jornadas Escolares Complementarias. Recuperado de https:/ /www.mineducacion.gov.co/1759/w3-article-235125.html

Mireles, P. (2017). El deporte como herramienta de integración social. (Tesis de grado, Universidad la Rioja). Recuperado de https://biblioteca.unirioja.es/tfe e/ TFE002619.pdf

Ordóñez, A. F., Polo, B., Lorenzo, A., \& Shaoliang, Z. (2019). Effects of a School Physical Activity Intervention in Preadolescents. Apunts. Educación Física y Deportes, 136, 49-61. doi:10.5672/apunts.2014-0983.es.(2019/2).136.04

Parra, O. J. (2003). La Educación en valores y su práctica en el aula. Tendencias Pedagógicas, 8, 69-88. Recuperado dehttps://revistas.uam.es/tendenciaspedagogicas/ article/view/1830

Pérez, O. M., Ramos, I. O., \& Achón, Z. N. (2007). Aprendizaje y desarrollo humano. Revista Iberoamericana de Educación, 44(3), 1-9. doi:10.35362/rie4432244

Registro Único de Víctimas-RUV. (2018). Víctimas del conflicto armado. Recuperado de https:// www.unidadvictimas.gov.co/es/registro-unico-devictimas-ruv/37394

Rodríguez Rosado, J., Iglesias Fernández, Á., \& Molina López, J. (2020). Evaluación de la práctica de actividad física, la adherencia a la dieta y el comportamiento y su relación con la calidad de vida en estudiantes de Educación Primaria (Physical activity patterns, nutritional habits, and behaviours and their relation with. Retos, 38, 129-136. Recuperado de https://recyt.fecyt.es/index.php/retos/ article/view/73921/48067

Taylor, S., \& Bogdan, R. (2006). Introducción a los métodos cualitativos de investigación. Barcelona: Paidós.

Torres, M. E. (2012). Desarrollo humano a través de la educación física en la escuela. (Tesis de grado, Universidad San Buenaventura Bogotá, Colombia). Recuperado de http://biblioteca.usbbog.edu.co:8080/Biblioteca/BDigital/ 66171.pdf

Valderrama, R., Solis-Espallargas, C., Trigueros, G., Manjón, J., \& Limón D. (2015). El deporte para todos como propuesta educativa para la inclusión y sustentabilidad social. Revista Fuentes, 16, 199-222. Recuperado de https:/ /revistascientificas.us.es/index.php/fuentes/article/view/ 2369/2187

Valenzuela, G. J., \& Flores, F. M. (2012). Fundamentos de Investigación Educativa. Monterrey, México: Editorial digital del Tecnológico de Monterrey 